\title{
FTIR Analysis \& Dielectric Constant for Some Iron Bismuth Borate Glasses
}

\author{
Hossam Mohammed Gomaa ${ }^{1 *}$ and Ahmed Hatim Hamdy EL-Dosokey ${ }^{2,3}$ \\ ${ }^{1}$ Optical Branch, High Institute of Optics Technology, Egypt \\ ${ }^{2}$ Physics Department, Faculty of Science, EL-Fayoum University, Egypt \\ ${ }^{3}$ Physic Department, Faculty of science, Sirte University, Libya
}

Received: 制 September 21, 2018; Published: 眥 September 26, 2018

*Corresponding author: Hossam Mohammed Gomaa, Optical Branch, High Institute of Optics Technology, Cairo, Egypt

\begin{abstract}
Some of iron bismuth borate oxide glasses were prepared to form a series of glass samples contain a constant and a fixed amount of $\mathrm{Fe}_{2} \mathrm{O}_{3}$ whereas the $\mathrm{B}_{2} \mathrm{O}_{3}$ was replaced by $\mathrm{Bi}_{2} \mathrm{O}_{3}$, according to the formula $20 \mathrm{~mol} \% \mathrm{Fe}_{2} \mathrm{O}_{3}-(15+\mathrm{x}) \mathrm{mol} \% \mathrm{Bi} 2 \mathrm{O}-(65-\mathrm{x}) \mathrm{mol}$ $\% \mathrm{~B}_{2} \mathrm{O}_{3}$, where $0 \leq \mathrm{x} \leq 20$. The X-ray diffraction and FTIR Spectra showed formation of homogenous short-range order structures. The values of the refractive index were estimated using both FTIR date room temperature dialectic measurements are in a good agreement with each other. It is clear that the $\varepsilon$ increased with the increasing of $\mathrm{Bi}_{2} \mathrm{O}_{3}$ content.
\end{abstract}

Keywords: Dielectric; FTIR; Amorphous Materials; Static dielectric; Oxide Glasses; refractive Index

\section{Introduction}

Borate glasses contain transition metal ions are of high importance in science and technology, as solid electrolytes and electric sensors [1]. Oxide glasses, especially bismuth borate glasses, are considered as stable active ion hosts for practical applications such as optical and sensor devices, because of their high refractive index [2]. The intensive studies of rare-earth doped glasses lead to good understanding their optical properties, which in turn help to choose the host material and the emitters for lasers [3]. Glasses containing transition metal ions such as $\mathrm{Fe}, \mathrm{Co}, \mathrm{V}$, etc. consider as semiconductors [4,5]. Electronic transport is only observed in these materials if the transition metal ion is present in two distinct sites [4]. The transport properties of oxide glasses have been of interest for a long time because of their potential application in technology. The ability of Born to exist in both three and four coordinated environments and high strength of the covalent B-O bounds, imparts borates, the ability to form stable glasses [6]. This work aims to study the effect of introduce $\mathrm{Bi}_{2} \mathrm{O}_{2}$ on both the refractive index and Static dielectric constant for some iron bismuth borate glasses.

\section{Experimental Measurements}

The chemical formula 20 mol\% $\mathrm{Fe}_{2} \mathrm{O}_{3}-(15+\mathrm{x})$ mol\% $\mathrm{Bi}_{2} \mathrm{O}_{3}-$ $(65-\mathrm{x}) \mathrm{mol} \% \mathrm{~B}_{2} \mathrm{O}_{3}, 0 \leq \mathrm{x} \leq 20$, have been used to prepared some of iron bismuth borate glasses. All batches were melted using an electric muffle furnace, in crucibles of porcelain, for about 120 mints. Then, finally poured in air at room temperature, two copper plates were used to get high fast cooling process. Electric capacities for all samples were carried out by a computer-controlled Stanford LCR bridge model SR 720 at four fixed frequencies [0.1,1,10 and $100 \mathrm{kHz}$.] at room temperature. Philips X-ray diffract-meter, model (P.W.1390b - $\lambda=1.5406 \AA$ ) was used for X-ray diffraction measurements at angles between $2 \theta=10^{\circ}$ and $80^{\circ}$ in steps of 0.2 degree with an integration time $0.4 \mathrm{~s}$ per step.

\section{Discussion of Results and Calculations}

\section{X-Ray Diffraction}

X-ray diffraction XRD is very useful tool for identification the order-disorder transformation, therefore it was used to check the internal structures of the studied samples. XRD patterns for the all studied samples were exhibited in Figure 1, where all sample showed same behavior. Checking Figure 1 carefully the shape shows two broad peaks have different intensities. $1^{\text {st }}$ peak was positioned approximately at $2 \theta=27 \mathrm{o}$, this peak is of highest intensity. While the $2^{\text {nd }}$ peak which of lowest intensity was positioned at $2 \theta=44 \mathrm{o}$. By refer to the XRD database it can state that these peaks may due to XRD by both Bi and Fe cations. Such broad peaks may refer to some 
small crystallites embedded in an amorphous matrix. Generally, the absence of any sharp peak means the short-range order nature of the internal structure of the studied samples.

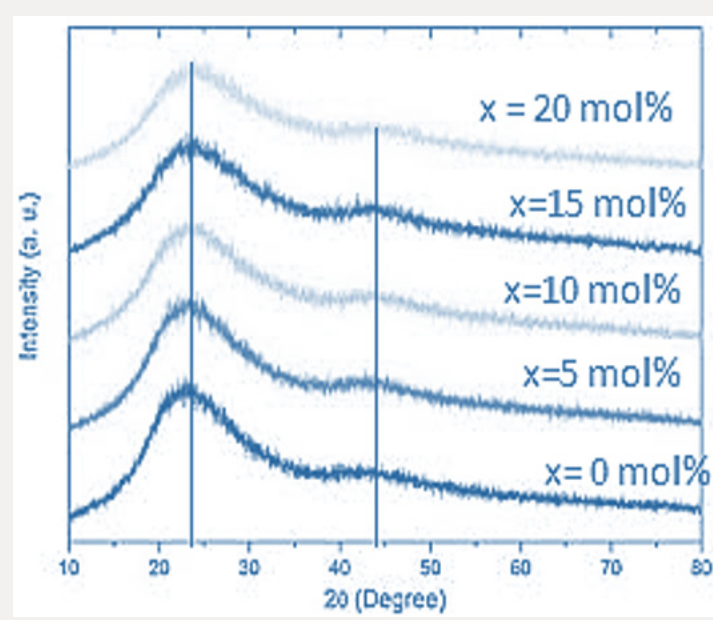

Figure 1: XRD for $\mathrm{x}=0$ and $20 \mathrm{~mol} \%$.

\section{FTIR Spectra Analysis}

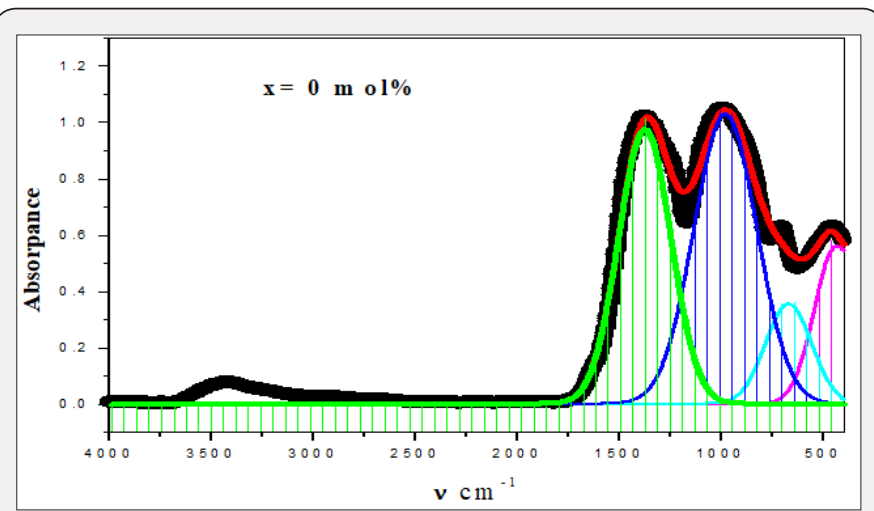

Figure 2: IR spectrum for $\mathrm{x}=0 \mathrm{~mol} \%$.

For a solid material, FTIR has an ability to characterize the order of the internal structure and building groups in addition to the chemical bonds. So, FTIR absorption spectra were recorded for all studied samples in range from $4000 \mathrm{~cm}^{-1}$ to $400 \mathrm{~cm}^{-1}$, as seen in Figures 2-6. Logically, it can observe that each spectrum contains multi-broad bands, each band may compose of more than one peak. Where each individual peak refers to a fixed type of vibration, which consequently characterize a certain bond or group unit. Such shapes characterize the solids own short range order structure, this observation is in an agreement with the XRD data. For more analytical study, each broadband was de-convoluted to some small peaks with different centers, then recorded in Table 1 where the band around $437 \mathrm{~cm}^{-1}$ may attribute to $\mathrm{Bi}-\mathrm{O}$ valence vibrations of very deformed BiO6 units [3-7] overlapping with Fe06 groups [8]. The relative area of this band increased as $\mathrm{Bi}_{2} \mathrm{O}_{3}$ was increased, the thing which may that $\mathrm{Bi}^{3+}$ have octahedral coordination and act as a glass network modifier and occupied only interstitial positions.
The center of this band has no observable change from sample to another, like observation may refer to the uniqueness of the structure network in all studied samples. Also, it may refer to an increase of the electric polarization, and hence increasing of the static dielectric constant. Band around $3500 \mathrm{~cm}^{-1}$ has a relative intensity increased as $\mathrm{Bi}_{2} \mathrm{O}_{3}$ was increased, like behavior may due to the ability of Bi cations to absorb water. The relative intensity of the band about $984 \mathrm{~cm}^{-1}$ refers to an increase of $\mathrm{BO}_{4}$ by increasing $\mathrm{Bi}_{2} \mathrm{O}_{3}$ content, while the bands about 686 and $1368 \mathrm{~cm}^{-1}$ refer to decease of $\mathrm{BO}_{3}$, like behavior may due to increase the fraction of the nonbridging oxygen atoms.

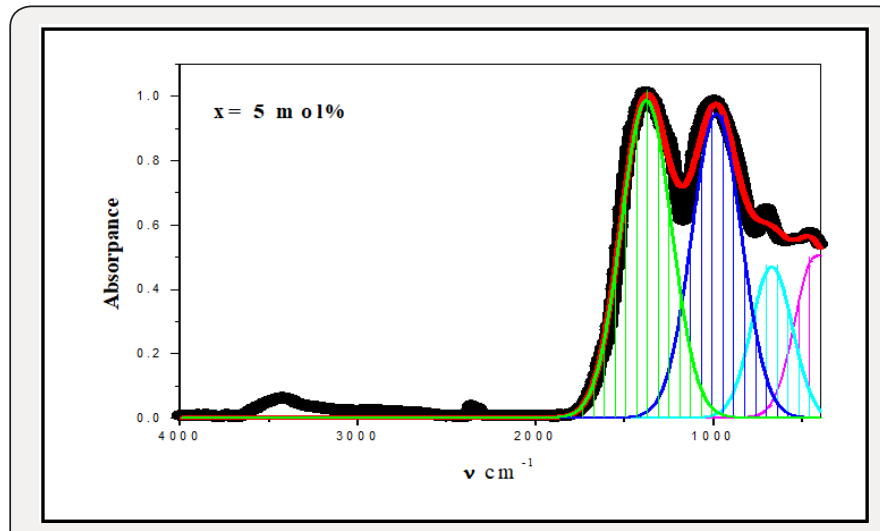

Figure 3: IR spectrum for $\mathrm{x}=5 \mathrm{~mol} \%$.

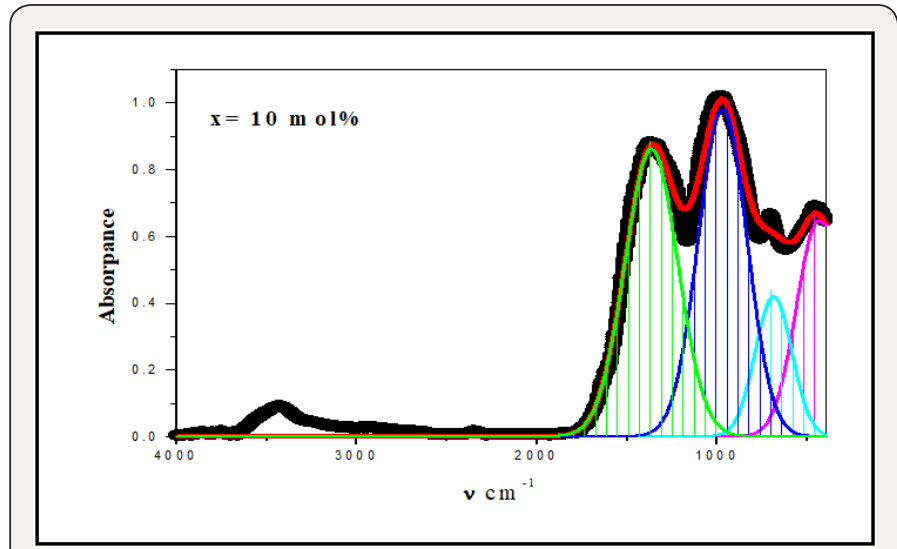

Figure 4: IR spectrum for $x=10 \mathrm{~mol} \%$.

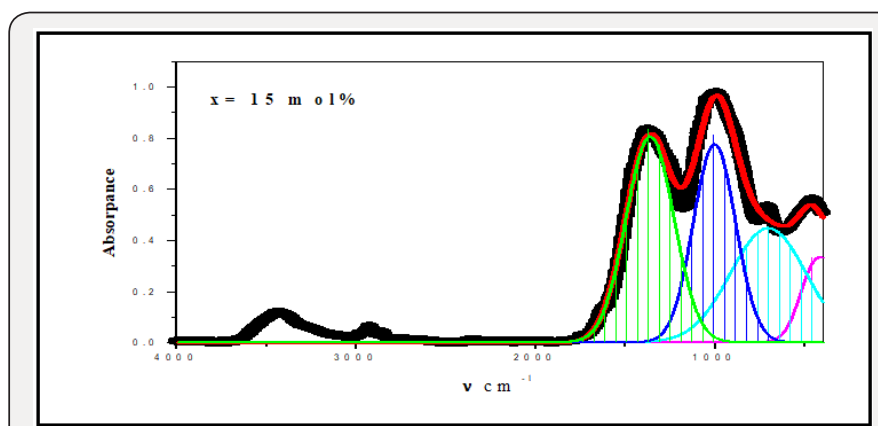

Figure 5: IR spectrum for $x=15 \mathrm{~mol} \%$. 


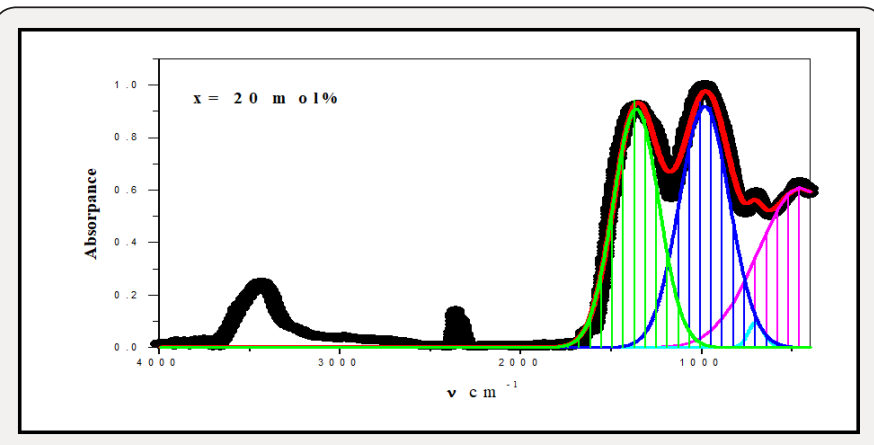

Figure 6: IR spectrum for $x=20 \mathrm{~mol} \%$.

Table 1: FTIR Data.

\begin{tabular}{|c|c|c|}
\hline Center & Assignments & Ref. \\
\hline $437\left(\mathrm{~cm}^{-1}\right)$ & $\begin{array}{c}\text { Bi-O valence vibrations of very } \\
\text { deformed } \mathrm{BiO}_{6} \text { units and/or the angle } \\
\text { modification of the B-O-B linkages/ } \mathrm{FeO}_{6} \\
\text { groups }\end{array}$ & {$[3-8]$} \\
\hline $686\left(\mathrm{~cm}^{-1}\right)$ & $\begin{array}{c}\text { the bending vibrations of B-O-B units in } \\
\mathrm{BO}_{3} \text { triangles }\end{array}$ & {$[1-10]$} \\
\hline $984\left(\mathrm{~cm}^{-1}\right)$ & Stretching vibrations of B-O in $\mathrm{BO}_{4}$ units & {$[9-3]$} \\
\hline $1368\left(\mathrm{~cm}^{-1}\right)$ & $\begin{array}{c}\text { Asymmetric stretching vibrations of B-O } \\
\text { in } \mathrm{BO}_{3} \text { units }\end{array}$ & {$[11]$} \\
\hline $\begin{array}{c}\mathrm{Above} \\
1550\left(\mathrm{~cm}^{-1}\right)\end{array}$ & Vibrations of OH groups & {$[1-11]$} \\
\hline
\end{tabular}

As an attempt to get all possible information from IR spectrum, Sellmeier equation [12], was resolved to obtain the average refractive index nFTIR for each sample, as seen in Table 2, Where $\lambda$ is the wavelength corresponding to one wavenumber (center of each observable peak).

$$
\begin{aligned}
& n_{i}=1+\frac{5.58245 \lambda^{2}}{\lambda_{i}^{2}{ }_{i}-(0.1625394)^{2}}+n_{i}=1+1+\frac{5.58245 \lambda^{2}}{\lambda_{i}^{2}-(0.1625394)^{2}} \\
& n_{\text {FTIR }}=\frac{\sum n_{i}}{\text { No.ofpeaks }}
\end{aligned}
$$

Table 2.

\begin{tabular}{|c|c|c|c|c|c|l|}
\hline $\mathbf{x ~ m o l} \%$ & $\varepsilon$ & & & & nave & \\
\hline & $0.1 \mathrm{kHz}$ & $1 \mathrm{kHz}$ & $10 \mathrm{kHz}$ & $100 \mathrm{kHz}$ & & $n_{\text {FTIR }}$ \\
\hline 0 & 12.5 & 3.5 & 3.3 & 3.2 & 2.25 & 2.69 \\
\hline 5 & 13.6 & 3.6 & 3.4 & 3.3 & 2.31 & 2.68 \\
\hline 10 & 14.7 & 3.8 & 3.6 & 3.4 & 2.38 & 2.68 \\
\hline 15 & 15.6 & 7.9 & 4.6 & 3.6 & 2.7 & 2.63 \\
\hline 20 & 16.6 & 8.5 & 5.3 & 3.7 & 2.8 & 2.65 \\
\hline
\end{tabular}

\section{Dielectric Constant}

The dielectric constant (relative permittivity), $\varepsilon$ of bulk solid may be known as the ratio of the capacitance of a certain capacitor contains this solid as a dielectric medium to the capacitance of the same capacitor with a vacuum as the dielectric medium. For the present work, for each sample, to determine the value of its dielectric constant at room temperature (RT), a piece of glass of uniform shape (has a fixed dimensions) was inserted as dielectric medium in the two parallel plates capacitor of LCR bridge. Figure 7 shows the dielectric constant $\varepsilon$ versus frequency at different $\mathrm{Bi}_{2} \mathrm{O}_{3}$ ratios. It is clear that the $\varepsilon$ increased with the increasing of $\mathrm{Bi}_{2} \mathrm{O}_{3}$ content, this may due to the increasing of the polarizing agents $\left(\mathrm{Bi}^{3+}\right.$ ions) in addition to the non-bridging oxygen atoms. Also, it is clear that $\varepsilon$ decreases by increasing the frequency, such behavior may due to the fact that as the frequency increases the orientational polarization decreases because the dipoles cannot follow the variation of frequency, so it takes more relaxation time than that of electronic and ionic polarization [13-15]. The following relation was used to obtain the values of the refractive index $\mathrm{n}$ as a function of the static dialectic constant, at only four fixed frequencies $0.1,1$, 10 and $100 \mathrm{kHz} .4$.

$$
\begin{aligned}
& n_{\text {ave }}=\sqrt{\varepsilon_{\text {ave }}} \\
& \varepsilon_{\text {ave }}=\frac{\varepsilon_{0.1}+\varepsilon_{1}+\varepsilon_{10}+\varepsilon_{100}}{4}
\end{aligned}
$$

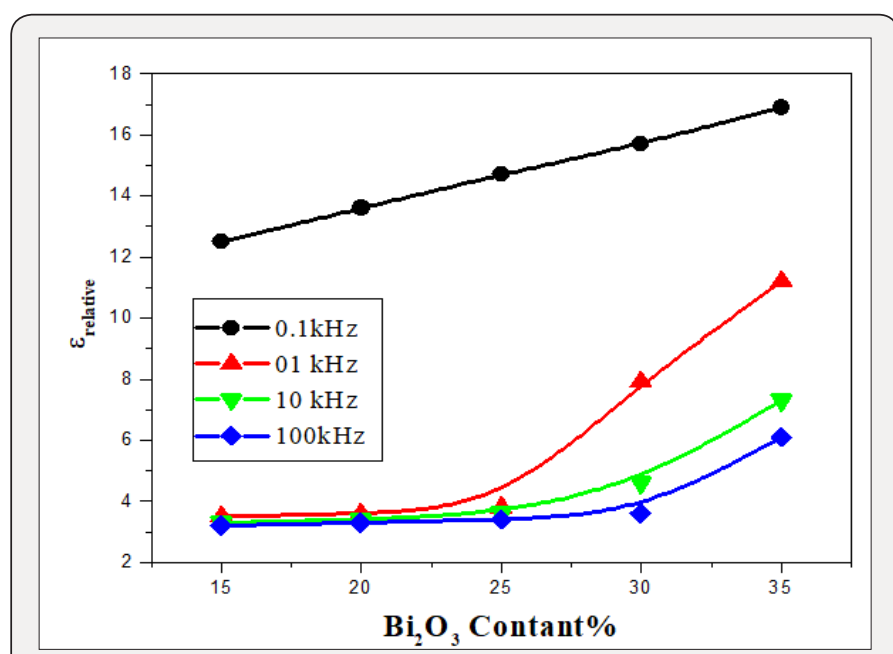

Figure 7: Dielectric constant.

\section{Conclusion}

In this study, the samples have prepared according to the chemical formula 20 mol\% $\mathrm{Fe}_{2} \mathrm{O}_{3}-(15+\mathrm{x})$ mol\% $\mathrm{Bi}_{2} \mathrm{O}_{3}-(65-\mathrm{x})$ mol\% $\mathrm{B}_{2} \mathrm{O}_{3}, 0 \leq \mathrm{x} \leq 20$. The X-ray diffraction and FTIR Spectra showed formation of homogenous short-range order structures. The calculated average refractive indices of samples estimated from IR spectra are in a good agreement with those calculated from the dielectric constant measurements.

\section{References}

1. KHS Shaaban, SM Abo-Naf, MEM Hassouna Physical and Structural Properties of Lithium Borate Glasses Containing $\mathrm{MoO}_{3}$. J of Silicon, p.1-8.

2. Ramesh Boda, MD Shareefuddin, MN Chary, R Sayanna (2016) FTIR and Optical Properties of Europium Doped Lithium Zinc Bismuth Borate Glasses. J of Materials Today: Proceedings 3(6): 1914-1922.

3. Yasser B Saddeek, K Alyn, Gh Abbady, N Afify, KHS Shaaban, et al. (2016) Optical and structural evaluation of bismuth alumina-borate glasses doped with different amounts of $\left(\mathrm{Y}_{2} \mathrm{O}_{3}\right)$. J of Non-Crystalline Solids 454: 13-18. 
4. GS Linsley, AE Owen, FM Hayatee (1970) Electronic conduction in vanadium phosphate glasses. J Non-Cryst Solids 4: 208-219.

5. AW Dozier, LK Wilson, EJ Friebele, DL Kinser (1972) J Am Ceram Soc 55: 37.

6. BS Rawal, Rk Maccrone (1978) J Non-Cryst Solids 28: 347.

7. P Venkateswara Raoa, G Naga Raju, P Syam Prasad, C Laxmikanth, N Veeraiah (2016) Transport and spectroscopic properties of nickel ions in $\mathrm{ZnO}-\mathrm{B}_{2} \mathrm{O}_{3}-\mathrm{P}_{2} \mathrm{O}_{5}$ glass system. J of Optik 127(5): 2920-2923.

8. I Kashif, H Farouk, AM Sanad, SA Aly, AM Assem (1992) Structural studies of some $\mathrm{V}_{2} \mathrm{O}_{5}-\mathrm{P}_{2} \mathrm{O}_{5}-\mathrm{B}_{2} \mathrm{O}_{3}-\mathrm{Fe}_{2} \mathrm{O}_{3}$ glass systems. J Material Science 27(1): 122-126.

9. S Cetinkaya Colak (2017) Role of titanium ions on the optical and thermal properties of zinc borate glass doped with $\mathrm{TiO}_{2}$. J of Glass Science and Technology Part B 58(2): 41-48.

10. I Kashif, A Ratep (2015) Role of copper metal or oxide on physical properties of lithium borate glass. J of Molecular Structure 1102: 1-5.
11. AA El-Maaref, KHS Shaaban, M Abdelawwad, Yasser B Saddeek (2017) Optical characterizations and Judd-Ofelt analysis of $\mathrm{Dy}^{3+}$ doped borosilicate glasses. J of Optical Materials 72: 169-176.

12. Thomas ME, Tropf WJ (1998) Infrared Refractive Index and Thermooptic Coefficient Measurement at APL. Johns Hopkins APL Tech Dig 19(3): 293-297.

13. Cornelius T Moynihan (1998) Description and analysis of electrical relaxation data for ionically conducting glasses and melts. J Solid State Ionics 105(1-4): 175-183

14. SS Fouad, AE Bekheet, AM Farid (2002) Derivation of a relation between the conduction mechanism and chemical bonding of amorphous $\mathrm{Ge}_{15} \mathrm{Se}_{85-x} \mathrm{Ag}_{x}$ alloys. J Physica B 322(1-2): 163-172.

15. GM Krishna, N Veeraiah, N Venkatramaiah, R Venkatesan (2006) Induced crystallization and physical properties of $\mathrm{Li}_{2} 0-\mathrm{CaF}_{2}-\mathrm{P}_{2} \mathrm{O}_{5}: \mathrm{TiO}_{2}$ glass system Part I. Characterization, spectroscopic and elastic properties. J Alloys and Compounds.

\section{(c) \\ This work is licensed under Creative Commons Attribution 4.0 License}

Submission Link:

$$
\text { Submit Article }
$$

DOI: 10.32474/DDIPIJ.2018.02.000139

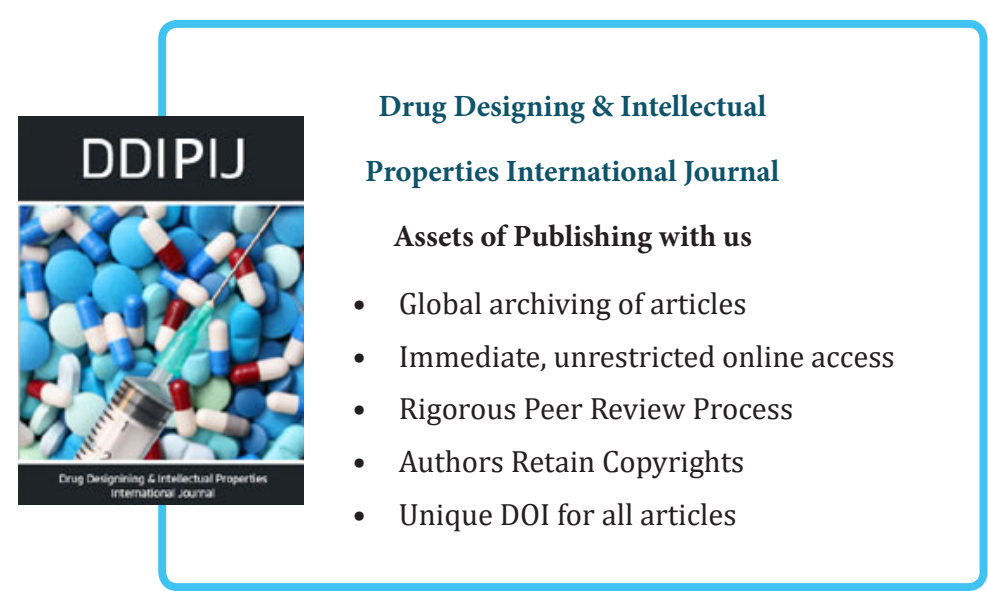

